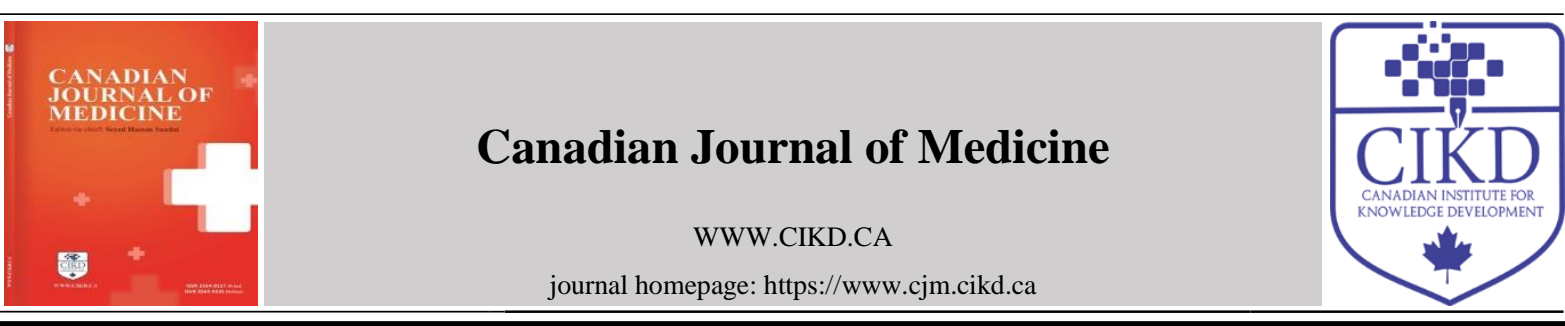

\title{
NSAIDs/Nitazoxanide/Azithromycin Immunomodulatory Protocol Used in Adult, Geriatric, Pediatric, Pregnant, and Immunocompromised COVID-19 Patients: A Real-World Experience
}

\author{
Mina T. Kelleni* \\ Pharmacology Department, College of Medicine, Minia University, Egypt
}

\section{Keywords:}

COVID-19, NSAIDs,

Diclofenac potassium,

Ibuprofen, Nitazoxanide,

Azithromycin, Telemedicine

\section{Received}

02 July 2021

Received in revised form

06 July 2021

Accepted

07 July 2021

*Correspondence:

mina.kelleni@mu.edu.eg

\begin{abstract}
COVID-19 management still lacks a protocol of proven efficacy, and we present a novel COVID-19 immunomodulatory protocol based on our early pioneering article, re-purposing nitazoxanide/azithromycin combination for early COVID-19 diseases. Our findings were followed by two articles to justify the addition of non-steroidal anti-inflammatory drugs to nitazoxanide/azithromycin. Furthermore, another recent article of ours illustrated the potential immunomodulatory mechanisms by which all the drugs used in this manuscript might be beneficial for COVID-19 patients. We presented a case series of 34 confirmed and highly suspected COVID-19 patients. It is noteworthy that 13 PCR-confirmed COVID-19 patients were included while the others were diagnosed by other measures and all cases were managed by telemedicine. The patients included adult males and females as well as children. All patients have received a short 5-day-regimen of NSAIDs / nitazoxanide/ azithromycin + - cefoperazone either in full or in part. The primary endpoint of this protocol was a full relief of all debilitating COVID-19 clinical manifestations, and it was fully achieved within two weeks. Most of the patients who were treated early have fully recovered during their described five days; the leucocytic/lymphocytic counts were significantly improved for those with prior abnormalities. Neither significant adverse effects nor post/para COVID-19 syndrome was reported. In conclusion, we present a pioneering 5-day protocol for the safe and effective treatment of COVID-19 using economic FDA-approved immunomodulatory drugs. We recommend conducting double-blind, randomized clinical trials with sufficient strength at the earliest opportunity.
\end{abstract}


Coronavirus disease 2019 (COVID-19) is one of the worst pandemics in modern history regarding its rapid rate of spread which has globally infected over 183 million and five hundred thousand individuals, and its mortality has been recorded to be approximately four million lives as of the $2^{\text {nd }}$ of July 2021. It should be taken into consideration that a vaccine might not be the ultimate safe, rapid, fair, or durable solution to compete with the huge global morbidity and mortality caused by the highly evolving severe acute respiratory syndrome Coronavirus 2 (SARS CoV-2) [1-3]. Therefore, we have searched for effective drugs that might be beneficial for the treatment of COVID-19. In April 2020, we published a pioneering manuscript that offers the scientific pathophysiological and pharmacological concepts for re-purposing a new, inexpensive, and relatively safe combination; nitazoxanide/azithromycin, for the early management of COVID-19 attributed to their potential effect in restoring and/or augmenting body's interferon homeostasis, when only eight clinical trials with recruitment difficulties had been registered for the potential of nitazoxanide in COVID-19 patients in developing countries [4].

In May 2020, a preprint was released [5] to suggest adding non-steroidal anti-inflammatory drugs (NSAIDs) such as ibuprofen for the early management of COVID-19. The important point in this preprint which was later updated, reviewed, and published in a reputable journal [6], is our suggestion that NSAIDs will be of great value to COVID-19 patients not only as safe and more effective analgesic and antipyretic drugs but also as potential players in reversing the pathogenesis of COVID-19, mitigating or preventing its complications and improving the clinical outcomes due to their potent anti-inflammatory and immunomodulatory effects, especially if used as early as possible during COVID-19. We relied on our early academic and real-life clinical experience which has evolved and we recently published other potential molecular protective mechanisms [7]. Furthermore, we claimed NSAIDs superiority over related advertised breakthroughs of low-dose aspirin [8] and colchicine [7].

Unfortunately, we have encountered medical malpractice, and misuse of corticosteroids [9], highly potent antibiotics as linezolid and anticoagulants in the management of mild-moderate COVID-19 patients that sometimes costed the lives of young patients, to be noted that those physicians are confident of impunity in the developing countries as the drug-induced mortality is falsely attributed to COVID-19. This study aimed to evolve a safe, effective, and targeted COVID-19 potentially curative protocol.

\section{Methods}

\section{Using Telemedicine to Prospectively Manage a Case-series}

This manuscript represented a telemedicine 34-case series (33 Egyptian patients living in Egypt or KSA and one citizen of Jordan) as part of our prospective observational study that reflected our academic and real-life experience that evolved a novel immunomodulatory COVID-19 management protocol. We managed over 50 COVID-19 cases through telemedicine. Yet, we opted not to repeat many of the non-PCR documented cases, and we have not presented many other cases that were managed through the traditional examination method. However, all share the same positive results. As early as late April/early May 2020, while we were working in KSA in isolated conditions away from our Egyptian patients and even when we have returned to Egypt and adopted our traditional medicine practice, we still receive some telemedicine requests. As a rule, we practiced telemedicine for the management of COVID-19 patients who 
could not appear for the traditional face to face, or mask to mask, physical examination because of conditions which prevented both domestic and international travel and the reasons also included other economic and COVID-19 related restrictions. The represented case series included adult males and females, as well as pregnant patients and children.

We have adopted a recommendation for de-identification regarding their age and occupation; hence, we have deleted their specific age and professions, yet we are proud that many of them were healthcare professionals.

\section{Diagnosis of COVID-19}

PCR confirmed thirteen patients, including a pregnant one, some patients have performed CT that showed ground glass opacities suggestive of COVID-19, few patients showed positive SARS CoV-2 IgM while the other cases have presented a combination of some or all of: 1history of close contact to first-degree relatives/ close associates COVID-19 patients who were confirmed by PCR. 2- clinical manifestations suggestive of COVID-19 e.g., fever, cough, dyspnea, anosmia, and ageusia. 3- laboratory investigations e.g., CBC of several patients showed leucopenia or lymphocytopenia, while CT, SARS CoV-2 IgM test or PCR test was neither medically justified, readily available, nor economically feasible for many of our patients. Therefore, we never asked for CT or PCR to guide our diagnosis, while we have early advised testing for SARS CoV-2 IgM in few cases, and later we have avoided it.

\section{COVID-19 Spectrum of Manifestations}

We have responded immediately to the received requests from the mentioned patients, and we used a personalized documented approach mostly via telemedicine using audiovisual social media applications, to be noted that properly adapted telemedicine has been rapidly evolving during this COVID-19 pandemic era [10]. Importantly, the clinical picture of COVID-19 was sometimes unusual, and this was of clinical importance to suspect early and to ask for investigations and management on an individualized basis. We agree to name a plethora of COVID-19 presentations as COVID-19 spectrum, which included the following: bizarre oral ulcers, oral lichen planus, concomitant herpetic labialis, unusual menstrual irregularities, unusual allergic dermatitis or skin rash, and suspected COVID-19-associated vasculitis. Many of our patients showed lymphopenia, while some have shown normal or marginally normal complete blood count but showed the history and clinical manifestations linked to COVID-19. Importantly, there were no restrictions on the management of COVID-19 patients, and some patients had moderate to severe COVID-19 manifestations, while many had several comorbidities, including obesity and diabetes mellitus. However, we professionally refused to manage some severe cases because of a lack of direct contact with the patients and a lack of close follow-up guarantee. We have insisted on contacting only the case/legal guardian, yet we accepted the consent coming from the husband and sons of a deteriorating patient who could not initially provide consent.

\section{Managing Adult Patients}

We have used the described a 5-day-protocol using NSAIDs, nitazoxanide (500 mg b.i.d. orally), azithromycin (500 mg q.d. orally) +/- parenteral cefoperazone as described in the manuscript. The protocol was used either fully or partly (Figure 1). 


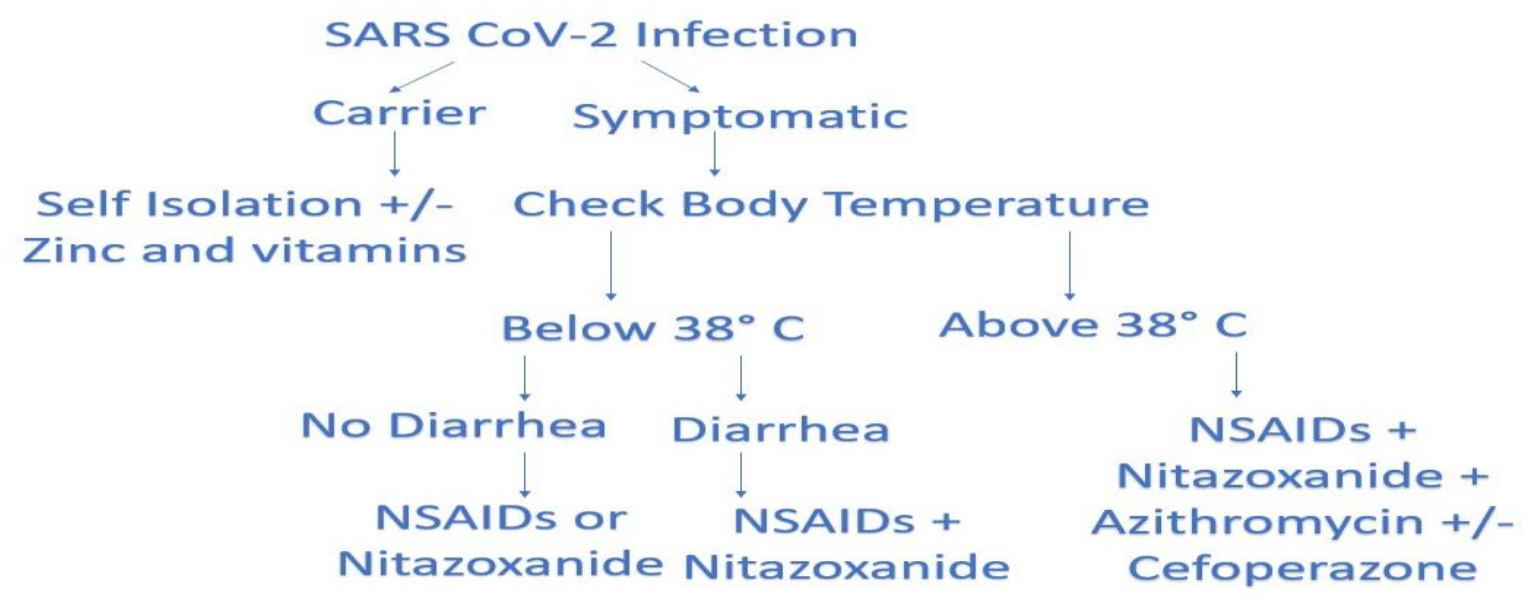

Figure 1. A novel protocol formanaging SARS CoV-2 positive cases.

We used oral postprandial NSAIDs, either diclofenac potassium $50 \mathrm{mg}$, ibuprofen (mostly $400 \mathrm{mg}$ ), lornoxicam $8 \mathrm{mg}$, or celecoxib 100 or $200 \mathrm{mg}$ b.i.d. alone for early cases complaining of sore throat, dry cough, or mild dyspnea with no fever or fever less than $38^{\circ} \mathrm{C}$. We observed that the earlier the administration, the more prompt the recovery of COVID-19. In addition, we used parenteral ketorolac $30 \mathrm{mg}$ in selected cases, as described in the manuscript. Importantly, we preferred to administer diclofenac twice daily, aiming to improve the natural immune response, if the patients recorded temperature less than $38^{\circ} \mathrm{C}$ in between the two doses, but they were permitted to receive it t.d.s. if the temperature exceeded $38^{\circ} \mathrm{C}$ before the next dose.

We used nitazoxanide with or without NSAIDs for those patients complaining of mild sore throat, diarrhea without fever, or fever less than $38^{\circ} \mathrm{C}$. As a rule, it was always given whenever diarrhea was reported. Notably, we have used nitazoxanide alone, if available in the pharmacies, as it is not in some countries, including KSA in which five of our PCR-confirmed patients resided, to manage mild COVID-19 patients who complained of GIT manifestations but did not complain of fever.

We usually prescribed the whole NSAIDs (as described)/ nitazoxanide /azithromycin fiveday protocol for COVID-19 adult patients complaining of fever more than $38^{\circ} \mathrm{C}$. Notably, we used personalized medicine to manage all cases, including one immunocompromised patient.

\section{Managing Pediatric and Pregnant Patients}

We prescribed the weight/age-adjusted doses of nitazoxanide suspension, azithromycin suspension, and ibuprofen syrup according to the same conditions described for adults and we have fully described our personalized approach to manage three pregnant COVID-19 patients. However, a 3-day-course of azithromycin and/or nitazoxanide was prescribed in pediatric patients with suspected mild COVID-19

The primary endpoint of our protocol was a full relief of COVID-19 debilitating manifestations e.g., fever, progressive cough, moderate/severe dyspnea and/or disturbed level of consciousness) as recovery from anosmia and ageusia was variable.

\section{Ethics, Consent, and Permissions}

This research was carried out according to the Helsinki declaration. We claim that compelling and purely scientific methodological reasons have led us to develop and use this protocol when 
there is no proven COVID-19 intervention. Our protocol provides a safe, effective, economic, and lifesaving one. According to the oath which we made when graduated from a college of medicine, we could not ignore to respond to SOS urgent requests from patients or to wait for ethical approval to save lives. Notably, our protocol had a potentially significant benefit and minimal risk when compared to some standard care of repurposed drugs/protocols [9, 11-13] and verbal informed consent was obtained from all patients, while we adopted strict international guidelines to protect patients' anonymity.

\section{Cases}

\section{Managing PCR Confirmed Adult COVID-19 Patients and their Close Contacts}

\section{Case 1}

The first case in the paper is a young man, in early 20s of age, who complained of fever (up to $38.8^{\circ} \mathrm{C}$ ), rhinorrhea, cough, headache, diarrhea, bone pain, anosmia, and ageusia. His complete blood count (CBC) revealed a total white blood cell count of $7.8 \times 10^{3} / \mu 1$, absolute lymphopenia with a relative lymphocyte count of $5.5 \%$, mixed population of monocytes, basophils, and eosinophils count of $16.5 \%$, and neutrophil count of $78 \%$. The findings revealed a positive RT-PCR test for SARS CoV-2 on the patient's throat swab; thus, we prescribed our ibuprofen/nitazoxanide/azithromycin protocol for 5 days. A significant clinical improvement was observed, and his total white blood cell count reached 6.3 X 103/ $\mu 1$, lymphocyte count reached $43 \%$, while the mixed population of monocytes, basophils, eosinophil count decreased to $8 \%$, and neutrophils count to $49 \%$.

\section{Case 2}

A man, in mid 50s of age, suffered from a mild cough, fatigue, and some colleagues prescribed him a course of azithromycin, an injection of betamethasone, and an antitussive/expectorant syrup containing oxomemazine, guaifenesin, sodium benzoate, and paracetamol. His condition was aggravated by fever up to $39^{\circ} \mathrm{C}$, marked cough, and severe malaise. Another colleague prescribed injection of ceftriaxone $1 \mathrm{~g}$ once daily, paracetamol $1000 \mathrm{mg}$ every 6 hours, and cough medicine for five days, resulting in partial improvement. He was therefore advised to test for COVID-19, where his PCR test was positive. A colleague then recommended increasing the dose of ceftriaxone every 12 hours and adding levofloxacin $500 \mathrm{mg}$ once daily but the patient decided to consult with us. We started our 5-day-regimen with parenteral cefoperazone $1 \mathrm{gm}$ once daily, azithromycin $500 \mathrm{mg}$ once daily for five days, and replaced paracetamol with lornoxicam $8 \mathrm{mg}$ once daily along with follow-up of the temperature. Furthermore, we stopped the administration of antitussive/expectorant syrup and replaced it with an antitussive supplement consisted of aqueous guava leaves extract and tilia flowers extract powder to be administered in days when severe episodes were encountered until his cough improvement. A marked improvement was seen in his condition, where $\mathrm{CBC}$ revealed a normal relative lymphocytic count of $36.2 \%$ four days after finishing our regimen as compared to $16.5 \%$ before treatment.

\section{Case 3 and 4}

A man, in early 30 s of age, complained of a mild fever of $37.8^{\circ} \mathrm{C}$, moderate malaise, anosmia and a positive RT-PCR test for SARS CoV-2 confirmed COVID-19. At the end of the 5-day 
course of azithromycin, he reported ageusia, cough, diarrhea, and malaise without fever and consulted us. We have successfully prescribed nitazoxanide and diclofenac potassium to manage his condition.

Interestingly, he also shared the illness of grandmother, in early 70s of age, who is known as a patient with controlled hypertension, suffering from cough, abdominal colic, and malaise. A tropical medicine consultant has asked for a $\mathrm{CT}$ chest that revealed ground-glass opacities suggestive of COVID-19; CBC showed a total white blood cell count of $3.3 \times 10^{3} / \mu 1$ and relative lymphocyte count of $13 \%$. The consulted colleague prescribed parenteral ceftriaxone, enoxaparin, paracetamol, zinc, and vitamin $\mathrm{C}$ with little improvement in the clinical picture when we were consulted. We stopped the use of enoxaparin and paracetamol but the use of zinc and vitamin $\mathrm{C}$ was continued. Furthermore, a 5-day diclofenac potassium and nitazoxanide course was prescribed, resulting in a dramatic improvement of her condition. A subsequent CBC showed a total white blood cell count of $5.5 \times 10^{3} / \mu 1$ and a relative lymphocyte count of $40 \%$.

\section{Case 5}

A young female citizen of Jordan, in early 30s, has suffered from fever up to $38^{\circ} \mathrm{C}$ (on paracetamol), headache, severe bone pain, and dizziness. A PCR test revealed positive COVID19. She was advised to self-isolation and administration of zinc and vitamin C. Upon consultation, we replaced paracetamol with naproxen $500 \mathrm{mg}$ once daily for five days and recommended azithromycin $500 \mathrm{mg}$ once daily. A dramatic improvement was revealed from the first day, and complete recovery was seen within five days.

\section{Case 6}

A hypertensive and type 2 diabetic man, in mid 40s of age, complained of troublesome fortnight dyspnea, dry cough and was quarantined after a nasopharyngeal and oropharyngeal swab showed a positive PCR test. Colleagues prescribed amoxicillin/clavulanate potassium/paracetamol regimen without improvement. He consulted with us, and we prescribed diclofenac potassium $50 \mathrm{mg}$ b.i.d. to replace paracetamol; no further antibiotics were prescribed as no fever was reported at the time of consultation. Interestingly, a significant improvement of his clinical manifestations was revealed from the first day when diclofenac potassium was administered, and he became mostly symptom-free in three days. The patient continued the 5day-regimen and then was discharged from a quarantine facility.

Notably, like other COVID-19 patients, we advised not to use antitussives for mild to moderate coughs and to use warm beverages, e.g., boiled mint, to soothe his sore throat. This approach has proved beneficial in all of our managed cases. However, this recommendation was based on a clinical sense that trusted the body's natural reflexes and was not based on a piece of evidence-based study.

\section{Case 7}

An adult COVID-19 man, confirmed by PCR, complained of a persistent mild to moderate cough, fluctuating fever in a quarantine facility for twenty days. We advised him to stop the previously prescribed antitussive dextromethorphan, and we prescribed diclofenac potassium/azithromycin. Notably, he reported that his persistent diarrhea improved 
immediately after stopping dextromethorphan. Furthermore, he has also used ibuprofen $600 \mathrm{mg}$ tablets, and a better clinical experience was reported compared with diclofenac potassium regarding headache, fatigue, and pain control. Finally, our patient was discharged after full recovery.

\section{Case 8 and 9}

A man, in late 50s of age, complained of $39^{\circ} \mathrm{C}$ fever associated with his SARS CoV-2 infection confirmed by PCR. We also prescribed diclofenac potassium/azithromycin and he received this treatment early and only zinc and vitamins were permitted to be concomitantly administered as he has already purchased them. His condition was reported to be fully controlled within the five-day-regimen.

Similarly, a man, in early 40 s of age, complained of fluctuating fever (up to $39^{\circ} \mathrm{C}$ ) which was not relieved by paracetamol, with a marked dry cough and COVID-19 diagnosis was confirmed by PCR. A colleague has prescribed azithromycin $500 \mathrm{mg}$ daily for three days and paracetamol. On the third day he consulted with us, we instructed him to continue azithromycin for another two days, and replaced paracetamol with diclofenac potassium for five days (t.d.s. for two days and b.i.d. for three days). A marked improvement in his clinical condition was reported, with normal temperature and gradual improvement of cough during the five days course.

\section{Case 10 and 11}

A young married couple consulted with us; the husband complained of fever, dry cough, fatigue, sweating, malaise, and dysgeusia, which was not relieved by paracetamol. PCR confirmed COVID-19 disease for this patient who was isolated at home. We prescribed diclofenac potassium/azithromycin to manage his condition, as well as zinc supplements. A marked improvement was obtained on the fifth day, but excessive sweating and mild fatigue persisted for five other days. Interestingly, his wife developed COVID-19 suggestive manifestations, e.g., fever, cough, malaise, severe sore throat, anosmia, and ageusia, only after an early PCR test revealed negative results. She received the same treatment as her husband with a similar dramatic improvement over five days.

\section{Case 12 and 13}

A man, in early 50s of age, has suffered from mild-moderate cough, fever of $37.5^{\circ} \mathrm{C}$, mild diarrhea and diaphoresis. A colleague prescribed azithromycin, oral acetylcysteine, cloperastine, zinc, and vitamin C. He experienced partial improvement, and his PCR test was found to be positive when he decided to consult with us. We advised the patient to continue azithromycin for five days and administered nitazoxanide b.i.d. for five days while stopped acetylcysteine and cloperastine. Pre-treatment laboratory investigation showed leucopenia (3.5 $\left.\mathrm{X} 10^{3} / \mu \mathrm{l}\right)$ and reduced absolute lymphocytic count $\left(0.97 \times 10^{3} / \mu \mathrm{l}\right)$ while his relative lymphocytic count was found to be $27.5 \%$, yet a normal $\mathrm{C}$ reactive protein (CRP). However, his D-dimer was mildly elevated $(0.67 \mathrm{mg} / \mathrm{l})$. We also prescribed meloxicam $7.5 \mathrm{mg}$ once daily for five days which started on the third day of the nitazoxanide course. Marked improvement was reported during the five-day regimen, and a CBC performed on the fifth day of our protocol showed a normal leucocytic count $\left(6 \times 10^{3} / \mu 1\right)$ and normal absolute lymphocytic count $(2.1 \mathrm{X}$ 
$10^{3} / \mu 1$ ), while the patient showed a relative lymphocytic count of $35.8 \%$ and a normal D-dimer level of $0.12 \mathrm{mg} / \mathrm{l}$.

Interestingly, while he was recovering, he again consulted with us as his mother, in early 80s of age, suffered from a dry cough, sore throat, headache, and fatigue. He reported that she has already received azithromycin and paracetamol for two days and had no improvement. We have recommended to continue a 5-day course of azithromycin and replace paracetamol with meloxicam $7.5 \mathrm{mg}$ once daily for five days and marked improvement in the five-day regimen has been reported except for mild fatigue and anorexia, which were fully resolved within a week after treatment.

\section{Case 14 and 15}

A married couple, in late 50 s of age, with a BMI over $35 \mathrm{~kg} / \mathrm{m}^{2}$, and a history of controlled hypertension while using olmesartan/amlodipine/hydrochlorothiazide for five years contacted us. The husband complained of fever (up to $38.8^{\circ} \mathrm{C}$ ), headache, sore throat, dry cough, and severe malaise. His wife encountered similar manifestations but without fever; CT was suggestive of COVID-19, and PCR also confirmed their conditions. We prescribed for both diclofenac potassium b.i.d., azithromycin, and nitazoxanide for five days. The husband's fever has subsided to normal from the first day, and on the fifth day they have reported almost complete resolution of symptoms except for mild cough, that was fully relieved two days later. Notably, they have also reported that they initially preferred to inhale, once or twice daily for two days, boiled cloves' water vapor to relieve their cough. Interestingly, a preliminary observation that arose from a small study suggested that cycles of steam inhalation at temperature $55-65{ }^{\circ} \mathrm{C}$ might be beneficial in halting SARS-CoV-2 virus infection during its initial stage and possibly preventing further spread [14]. Other researchers demonstrated that SARS CoV-2, derived from various sources, is thermally inactivated, and re-purposing of domestic steam disinfectors was suggested [15]. We suggest that their recommendation is totally harmless and might be at least as beneficial as that of zinc and vitamins supplements.

\section{Case 16}

A pregnant patient confirmed SARS-CoV-2 infection by PCR consulted with us, and discussed in the following sections that demonstrate managing non-PCR confirmed COVID-19 adult patients, including one immunocompromised, two other pregnant patients and four children.

\section{Case 17}

An immunocompromised man, in mid 30s of age, who was receiving a long-term maintenance dose of azathioprine to control autoimmune central nervous system vasculitis, has been in close contact with a SARS CoV-2-positive patient. He complained of moderate fatigue, fever of $37.8^{\circ} \mathrm{C}$, mild cough, and rhinorrhea. His $\mathrm{CBC}$ showed a normal leucocytic count of $5.32 \mathrm{X}$ $10^{3} / \mu 1$ (normal range: $5-11 \times 10^{3} / \mu 1$ ), reduced relative and absolute lymphocytic count $(18.2 \%$ and $0.97 \times 10^{3} / \mu 1$ respectively), and he was self-prescribed with azithromycin, zinc, vitamin $\mathrm{C}$, and paracetamol. Two days later, repeated CBC revealed leucopenia $\left(3.8 \times 10^{3} / \mu 1\right)$ with an increase in both the relative and absolute lymphocytic count (40\% and $1.5 \mathrm{X} 10^{3} / \mu \mathrm{l}$ respectively), normal CRP, and D-dimer levels. However, he still complained of dizziness, lowgrade fever, headache, fatigue, and rhinorrhea and he decided to consult with us. Since he has 
already finished a 5-day-regimen of azithromycin, we only replaced paracetamol with lornoxicam $8 \mathrm{mg}$ b.i.d. for five days. The patient reported significant improvement in his clinical condition with relief of all symptoms. His CBC showed an improved normal leucocytic count $\left(4.1 \times 10^{3} / \mu 1\right)$ and relative and absolute lymphocytic count of $40 \%$ and $1.6 \times 10^{3} / \mu 1$ on the sixth day of our regimen.

\section{Case 18}

A female patient, in mid-30s of age, has suffered from productive cough and mild rhinorrhea. CT scan was suggestive of COVID-19 (Figure 2). A colleague has prescribed her ceftriaxone 1 gm (IV), followed by dexamethasone $8 \mathrm{mg}$ (IV), oral prednisone $5 \mathrm{mg}$ b.i.d., ketorolac (IV) once daily for five days. Furthermore, she also received clarithromycin $500 \mathrm{mg}$ daily for one week, ivermectin, levocetirizine, vitamin $\mathrm{C}$, zinc, acetylcysteine, erdosteine, and rivaroxaban for one week and her clinical condition was deteriorating as revealed by an increase in severity of cough and progressive fatigue. Upon consultation with us, and as she reported no fever before or during the previous treatment, we advised her to discontinue all drugs. The severe cough episodes and her clinical condition have gradually improved over one week. She only was advised to rest, healthy nutrition, warm beverages, and inhalation of boiled cloves' water vapor twice daily, and a full, smooth recovery was reported in another week. We wish to strongly warn against COVID-19 drug abuse and we claim that this drug abuse might have wasted more lives than that lost due to SARS CoV-2 complications.

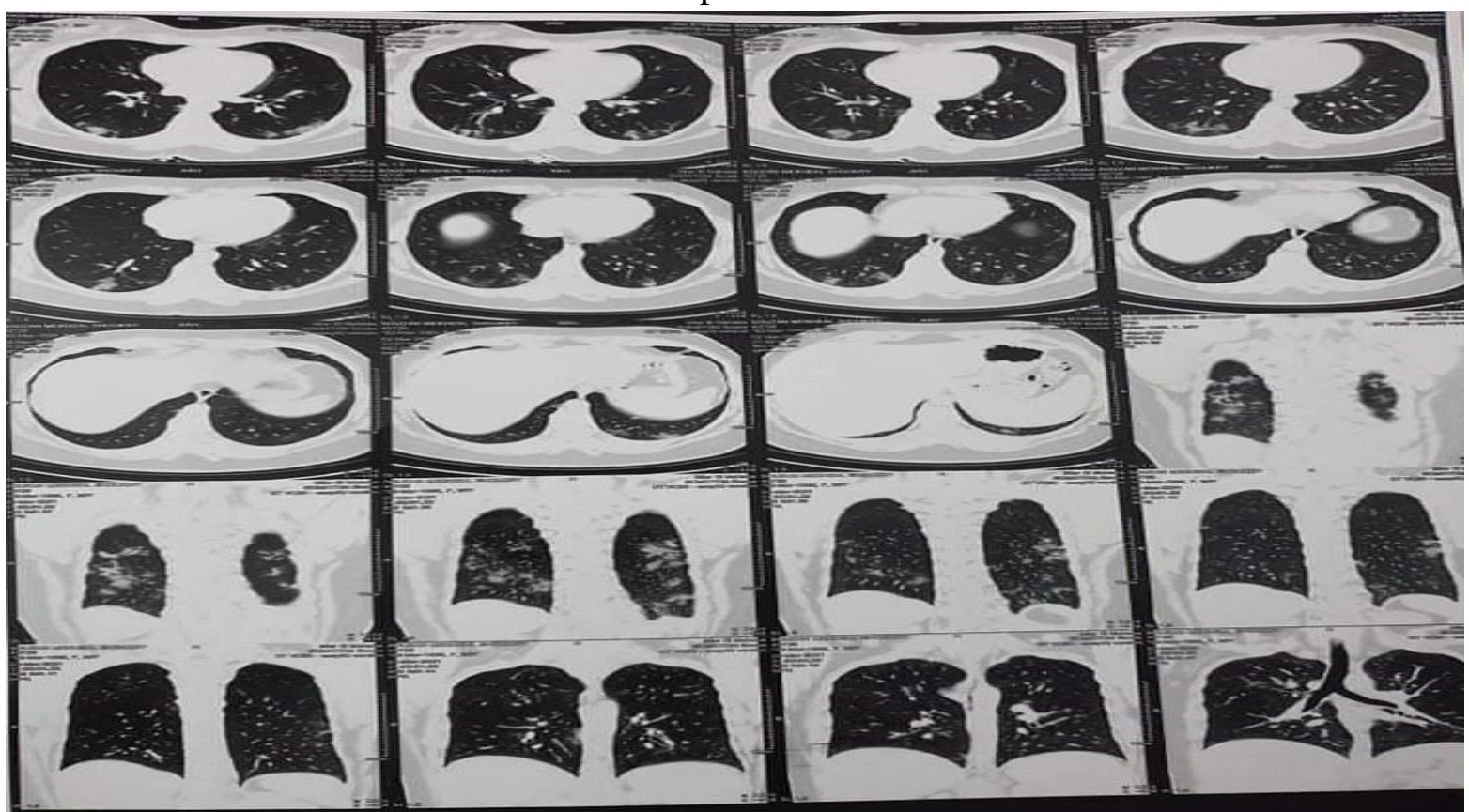

Figure 2. CT showed features of COVID-19 disease.

\section{Case 19}

A female patient, in mid-40s of age, consulted with us after her husband's isolation due to a COVID-19 suspicion. She reported fever up to $39.2^{\circ} \mathrm{C}$, sore throat, severe headache, and bone pain. She self-administered azithromycin, paracetamol, zinc, and vitamin $\mathrm{C}$ for one day without improvement. When consulted, we asked for laboratory investigations, where leucopenia (3.64 $\mathrm{X} 10^{3} / \mu 1$ and reduced relative and absolute lymphocytic counts $\left(15 \%\right.$ and $0.55 \mathrm{X} 10^{3} / \mu 1$ respectively) was found. We advised her to continue using azithromycin for five days and 
paracetamol was replaced with parenteral ketorolac on the first day of a 5-day-course of meloxicam (7.5 mg once daily). Marked improvement was reported on the first day of our regimen. A CBC follow-up on the $12^{\text {th }}$ day of our consultation showed a normal leucocytic count $\left(7.4 \times 10^{3} / \mu 1\right)$ and normal relative and absolute lymphocytic counts $\left(30 \%, 2.22 \times 10^{3} / \mu 1\right.$, respectively).

\section{Case 20}

A man, in mid-30s of age, was a close contact of a quarantined COVID-19 confirmed by PCR patient, and he suffered from deterioration of severe cough episodes, dyspnea and herpetic labialis that followed his self-prescribed two injections of cefotaxime (antibiotics can be readily purchased without a prescription in Egypt and several developing countries), zinc, and vitamins to manage his COVID-19 suggestive manifestations. Though fever was reported initially, at the time when he consulted with us, no fever was encountered. We prescribed lornoxicam $8 \mathrm{mg}$ b.i.d. for five days, with a ketorolac injection once in the first day, to control reported severe body pain, to safely manage his condition. He described a significant improvement in five days, yet residual mild-moderate cough, moderate-severer headache, malaise persisted for another week to almost full recovery. Notably, only water steam inhalation was advised to control his severe cough episodes as at the time of his management, our described pharmacotherapy for COVID-19 associated cough was not evolved as described in other cases.

\section{Case 21}

A female patient, in late 40s of age, complained of severe sore throat, malaise, fever and mild cough and her CT chest revealed patchy, scattered ground-glass opacities suggestive of COVID-19, as described in the given report, and a colleague has prescribed paracetamol, betamethasone injection IM, doxycycline $100 \mathrm{mg}$ q.d. for 15 days, azithromycin $500 \mathrm{mg}$ q.d. orally, some vitamins and minerals as well as a syrup that contains antihistaminic and expectorant for three days. Her condition rapidly deteriorated with persistent fever, yellow vision, tachycardia and marked confusion that prevented her from giving a consent. We accepted a compassionate request from her husband and sons to help and asked for an urgent CBC that revealed a total leucocytic count of $5.1 \times 10^{3} / \mu 1$ with a low lymphocytic count of $12 \%$. She was also diagnosed positive for SARS CoV-2 IgM test upon our request, a practice that we opted for initially in our practice and soon we abandoned it suggesting it is not necessary according to our safe management protocol. We stopped the previously prescribed antitussive syrup, as explained earlier and we also stopped the bacteriostatic doxycycline and prescribed azithromycin $500 \mathrm{mg}$ q.d., a ketoprofen IM injection in the first day to be followed by diclofenac potassium b.i.d. for five days. Furthermore, we added empiric parenteral cefoperazone; $1 \mathrm{gm}$ once daily during the first three days of our regimen, we have chosen it for its ready availability and its broad-spectrum antibacterial efficacy against atypical respiratory micro-organisms that was clinically suspected to share in the development of high fever and her deteriorating condition, as a superinfection. Furthermore, we have added nitazoxanide b.i.d. that also managed to control an episode of diarrhea that has been encountered after the start of treatment. Her condition improved gradually yet dramatically within five days and the CBC on the fifth day revealed an improved total leucocytic count of $6 \times 10^{3} / \mu 1$ with a significantly elevated 
lymphocytic count of $23 \%$. She returned almost normal within two weeks, complaining only of mild cough and insignificant chest tightness that have disappeared later spontaneously.

\section{Case 22 and 23}

A man, in mid 50s of age, with a history of type 2 diabetes, hypertension, obesity (BMI 33.2) and using glyburide/metformin, bisoprolol, and olmesartan/hydrochlorothiazide complained of cough, fever, $39^{\circ} \mathrm{C}$, and shortness of breath. His CT scan showed features of COVID-19 disease, and his CRP was found to be $76 \mathrm{mg} / \mathrm{l}$, followed by a marginally normal relative lymphocytic count of $24.6 \%$. A colleague has prescribed the mucolytics erdosteine and acetylcysteine, zinc, vitamin C, rivaroxaban $10 \mathrm{mg}$, prednisolone $20 \mathrm{mg}$, ceftriaxone $1 \mathrm{gm}$ (IV), dexamethasone $8 \mathrm{mg}$ (IV), diclofenac sodium (IV), and famotidine (IV) for eight days. This colleague has also prescribed ipratropium bromide inhalation sessions; however, these sessions were stopped quickly when his oxygen saturation worsened. His clinical condition was deteriorating as well as his oxygen saturation reached $80 \%$ without oxygen inhalation, while it was found to be $92 \%$ with oxygen. Furthermore, his clinical manifestation revealed to be worsened, e.g., severe cough episodes, dyspnea, increased fatigue, insomnia, fluctuating fever up to $38^{\circ} \mathrm{C}$, elevated blood glucose profile, and hypertensive episodes.

Upon consultation on the $9^{\text {th }}$ day, we continued the antihypertensive and anti-diabetic drugs, but prednisolone and all the parenteral medications, including dexamethasone, were stopped. We have also stopped the mucolytics in a trial to ameliorate the severe cough episodes and replaced zinc, vitamin C, and lactoferrin with ergocalciferol 10,000 IU q.d.

Moreover, we first tried to give an antitussive supplement containing aqueous guava leaves extract and tilia flowers extract but we shifted to the more potent cloperastine. Although the patient was not suffering from fever, a daily dose of azithromycin was prescribed for five days due to the reports of fluctuating fever. We added nitazoxanide b.i.d. and intentionally low-dose celecoxib (100 mg) once daily and advised a low potassium diet for five days and continued oxygen inhalation. The patient showed gradual improvement in five days and when the cough has decreased, he was finally able to sleep in the supine sleeping position and a $95 \%$ oxygen saturation without oxygen supplementation was reported. Furthermore, his blood pressure and blood glucose profile returned to the pre COVID-19 controlled normal measurements. Notably, we advised adding insulin glargine to control the persistently elevated blood glucose level, but the patient clinical condition was improved and did not need it. Interestingly, a cutaneous rash has appeared on the neck and back (Figure 3) on the fourth day of our regimen and we considered it a good sign of recovery. 


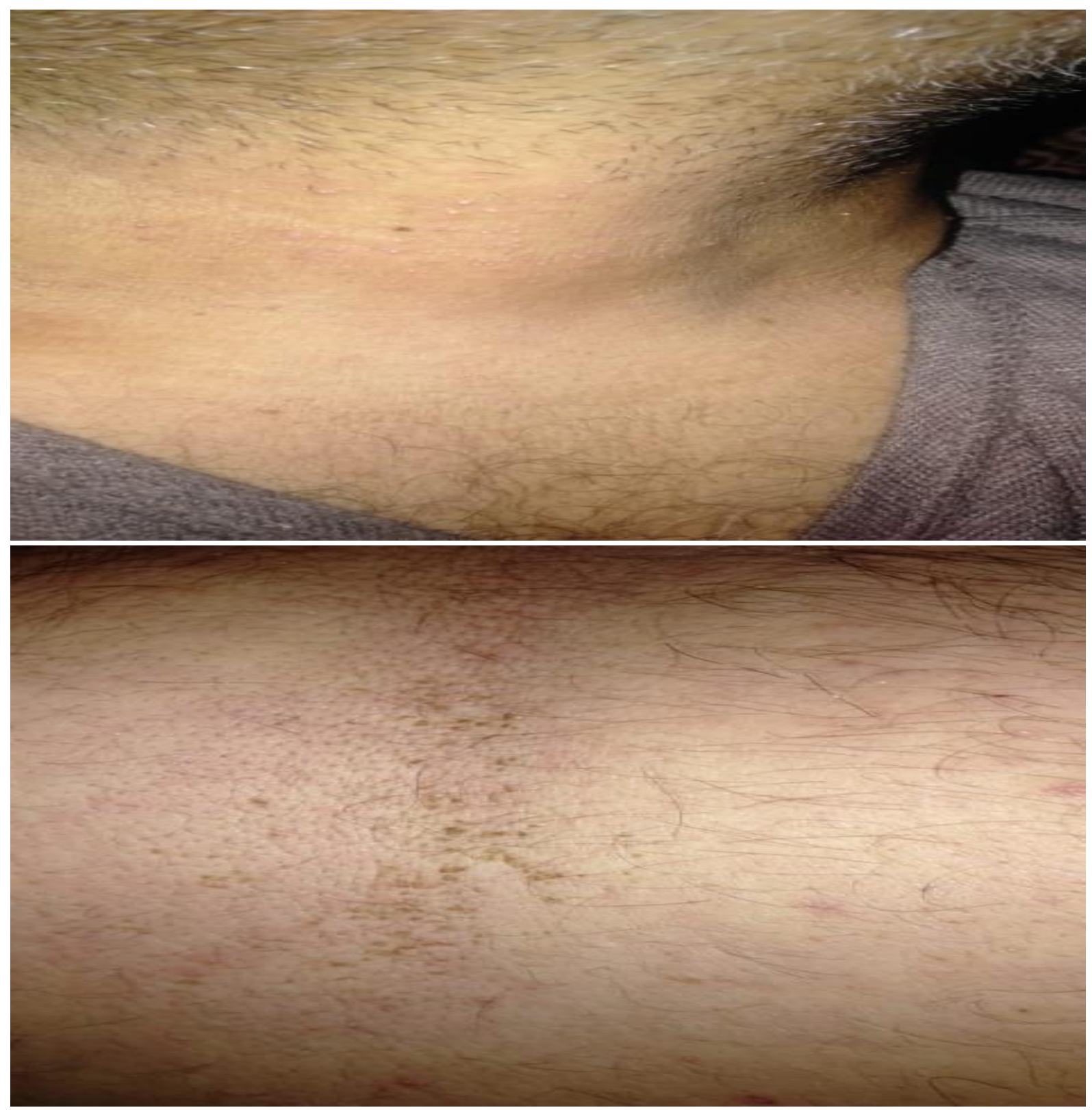

Figure 3. Suspected COVID-19 patient with an improvement of rash on the neck and back

However, he reported dysphagia and we suspected fungal infection, and he was markedly improved with oral nystatin t.d.s. for three days. Similarly, fungal laryngitis was suspected when dysphonia was reported, so we prescribed oral fluconazole $150 \mathrm{mg}$ once daily for ten days, during which the patient showed significant gradual improvement in both possible manifestations of the fungus.

Interestingly, CRP on the sixth day of our treatment has decreased to $12 \mathrm{mg} / \mathrm{l}$, and the lymphocytic count was elevated to $41 \%$. However, his D-dimer level has revealed an elevated $1 \mu \mathrm{g} / \mathrm{ml}$ level; thus, we prescribed a low dose $(100 \mathrm{mg})$ of aspirin once daily for one week, but the level has not decreased so we replaced it with apixaban $2.5 \mathrm{mg}$ b.i.d. for another week without adverse effects. However, we reevaluated the risk-benefit ratio due to the possible low association of D-dimer levels with venous thromboembolism in other inflammatory diseases [16], and thus we, thereafter, avoided prescription of direct oral anticoagulants to ambulatory patients with mild elevation D-dimer levels. Notably, we allowed a recovery without any of our 
protocol drugs starting from the sixth day for the remaining mild cough episodes and advised inhalation of boiled cloves' water vapor. The patient has reported a speedy recovery.

His daughter, in mid-20s of age, has concomitantly suffered from fever up to $38^{\circ} \mathrm{C}$, colic, anorexia, and fatigue. Her CRP was $12 \mathrm{mg} / \mathrm{l}$, and D-dimer was mildly elevated $(0.52 \mathrm{mg} / \mathrm{l})$. She reported ingestion of one tablet of ampicillin $500 \mathrm{mg} /$ flucloxacillin $250 \mathrm{mg}$. Thus, we advised completing this course t.d.s for three days in addition to azithromycin once daily and diclofenac potassium $50 \mathrm{mg}$ b.i.d. for five days. Notably, we added metronidazole $500 \mathrm{mg}$ b.i.d. for three days to decrease the incidence of antibiotic-associated colitis. She reported significant recovery starting from the second day with no recurrence in fever and significant improvement of the other manifestations. Only mild and self-limited diarrhea was reported on the second day of the five-day regimen, and she reported full recovery of all the original manifestations.

\section{Case 24}

A female patient, in early 20 s of age, complained of sore throat, fever, dyspnea, severe headache, marked malaise, and recurrent troublesome cough episodes. Our patient received azithromycin, paracetamol, zinc, and vitamin $\mathrm{C}$ with no improvement for two days. Her CBC showed leucopenia and a low positive titer of $\mathrm{C}$ reactive protein. Upon consultation, no fever was detected. Since azithromycin was already administered, we continued its administration and replaced paracetamol with celecoxib $200 \mathrm{mg}$ once daily for five days; the prescribed zinc was also changed to a lower one [17]. Moreover, marked clinical improvement regarding headache, cough and malaise was revealed from the first day of the five days course. Notably, celecoxib has been advised by other researchers to be clinically tested for COVID-19[18] and when celecoxib was used as adjuvant therapy, it was suggested to be capable of promoting the recovery of all types of COVID-19 and reducing the mortality rate of elderly and those with comorbidities [19]. Importantly, this patient complained of acute episodes of tachycardia and generalized abdominal colic that were started before our therapy and persisted for four days. We suspected COVID-19-associated vasculitis, and this potential COVID-19 complication was effectively managed by administering pentoxifylline $400 \mathrm{mg}$ b.i.d. for five days. Notably, pentoxifylline was reported to be effective in managing another type of immunoinflammatory induced vasculitis [20]. It was also suggested by other researchers to be beneficial as adjuvant therapy for COVID-19 [21].

\section{Case 25}

A man, in mid-40s of age, with a history of type 1 diabetes showed a clinical picture of mild to moderate COVID-19 along with fever $39^{\circ} \mathrm{C}$. A colleague has prescribed him azithromycin, paracetamol, zinc, and vitamin $\mathrm{C}$ for only three days. However, a more severe clinical picture of high fever, severe fatigue, profuse sweating, severe episodes of cough with newly developed dyspnea has developed after two days of stopping treatment. He consulted with us on that fifth day and a computed tomography confirmed viral pneumonia with bilateral scattered subpleural and parenchymal patches of ground-glass opacities, suggesting COVID-19 disease (Figure 4). We prescribed parenteral cefoperazone, q.d., for three days with another course of azithromycin (500 mg q.d.) for five days and oral diclofenac potassium, t.d.s., for the first two days, followed by twice daily for three other days when his condition started to improve. It is worth noting that we have not prescribed nitazoxanide for this patient. 


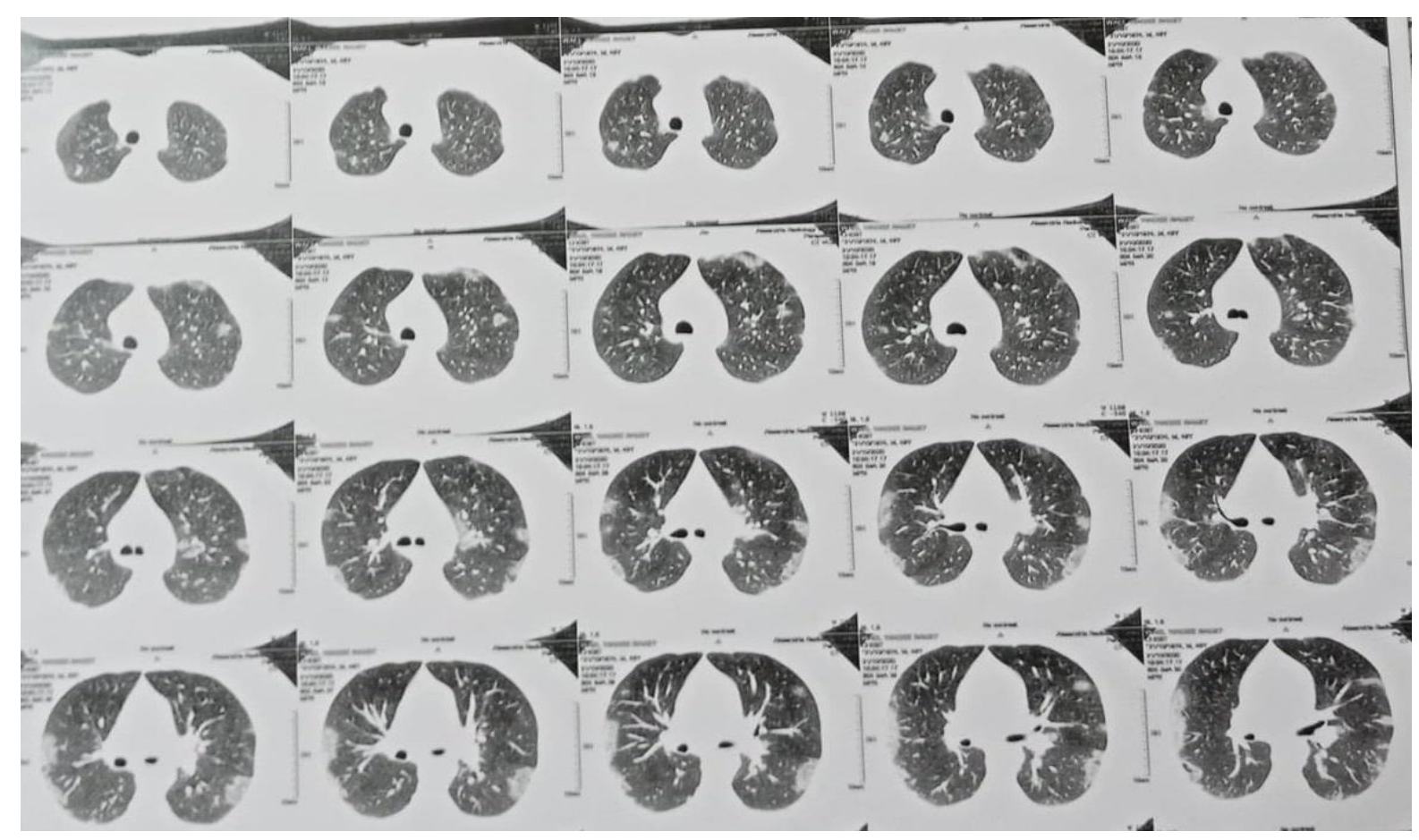

Figure 4. Pre-treatment chest CT suggestedCOVID-19 disease.

With the body temperature returning to normal without any antipyretic drugs or antibiotics after our 5-day course, the patient has experienced a significant clinical improvement in terms of most symptoms. He has also experienced gradual improvement of his severe cough and severe episodes after seven days without antitussive drugs and returned almost normal after two weeks. Interestingly, a follow-up CT was performed in another center one week after the previous one and revealed a regressive course of viral pneumonia with residual bilateral scattered patches of ground-glass opacities. Notably, the lymphocytic count of this patient appeared normal, $30 \%$ before treatment, but it has increased to $47.9 \%$ after the 5-day-course of treatment, raising a possibility that it might has been deteriorating; thus, the NSAIDs therapeutic intervention might had efficiently stopped and reversed this deterioration as was also suggested previously [6].

Importantly, The CRP level of this patient was $54 \mathrm{mg} / \mathrm{l}$ at the start of our treatment and was elevated to $90 \mathrm{mg} / \mathrm{l}$ at the end of the 5 -day-course, and its level has decreased to $9 \mathrm{mg} / \mathrm{l}$ one week after the course. Thus, the initial increase in CRP might be explained by an undergoing concomitant bacterial infection that has been treated efficiently by the administered antibiotics. Interestingly, this patient was suffering from type 1 diabetes mellitus for eight years, and he has experienced a significant deterioration of his blood glucose profile. Thus, daily biphasic isophane insulin dosage was increased, and an adjusted dose of insulin glargine was given for two weeks.

Notably, we have not been in direct contact with moderate-to-severe patients; thus pharmacovigilant low-dose regimen of cefoperazone was considered to avoid the scarce potential of vitamin K-dependent coagulopathy which might develop with serious diseases[22]. Cefoperazone was administered with a full 5-day-course of azithromycin. Interestingly, as the patients' clinical condition improved, no increase of cefoperazone dose or duration of therapy was required. 
Meanwhile, we recommended all the encountered patients to remain isolated for 21 days from the beginning of the symptoms, including ten days of a completely asymptomatic period to avoid the possibility of false negative PCR results. We know that our advice for the isolation period is not consistent with the mainstream of the current guidelines [23]. However, a study has recommended a 22-day quarantine period to avoid the $6.7 \%$ failure, where symptoms after the 14-day quarantine period were seen; the 22-day quarantine showed a failure rate below $1 \%$ with $95 \%$ confidence [24]. In addition, RT-PCR findings of 301 confirmedCOVID-19 patients showed the average contagious period of infected patients was 20 days [25].

\section{Managing COVID-19 Pregnant Patients}

Furthermore, the patients included three pregnant patients; one in her $9^{\text {th }}$ week of gestation, another highly suspected case in her $18^{\text {th }}$ week and a third one was in her $34^{\text {th }}$ week of gestation.

\section{Case 16}

A pregnant patient diagnosed as a COVID-19 patient in her $9^{\text {th }}$ week of gestation (confirmed by SARS CoV-2 rapid IgM test and PCR); in addition, she has also been treated for rheumatoid arthritis. Her COVID-19 clinical picture included fever, sore throat, fatigue, and dry cough and she consulted with us. We stopped both hydroxychloroquine and sulfasalazine, which have been already used for rheumatoid arthritis, during our azithromycin/nitazoxanide five-day treatment course, to prevent potential adverse drug interactions with our protocol. Notably, we have not prescribed NSAIDs as she was also already receiving a maintenance low dose of prednisone for her rheumatoid arthritis. Prenatal four-dimensional ultrasonography showed a healthy female fetus with no suspected congenital abnormalities, and she gave birth to a healthy newborn.

\section{Case 26}

Another pregnant patient was in her $18^{\text {th }}$ week of gestation and was highly suspected of COVID19, by a history of close contact to a positive COVID-19 case. The patient presented clinical manifestations of sore throat, cough, and declining lymphocytic titer (24\%) compared to a previous pre-COVID-19 CBC (39\%). We prescribed a five-day course of diclofenac potassium $50 \mathrm{mg}$ b.i.d. and she decided to add two more days. Her COVID-19 symptoms were relieved without significant adverse effects. She gave birth to a healthy female newborn. We suggest that the potential benefits might exceed the risks for this short-term NSAIDs regimen in pregnant patients before the third trimester. Moreover, we suggest that our adopted NSAIDs dose and duration of therapy is matching the recently updated requirements that the FDA has suggested regarding NSAIDs administration to pregnant patients at 20 weeks till the beginning of the third trimester to avoid oligohydramnios.

\section{Case 27}

A third pregnant patient was in her $34^{\text {th }}$ week of gestation, and complained of self-limited diarrhea, mild rhinorrhea, sore throat, headache, moderate dry cough, dyspnea, and a low-grade fever for one week. Her CBC was normal, but the erythrocyte sedimentation rate (1st hour) was positive $(42 \mathrm{~mm} / \mathrm{hr})$. A colleague has prescribed levofloxacin for this patient, and she has received the first dose. Her husband simultaneously complained of a mild cough. 
Upon consultation, we stopped levofloxacin and replaced it with azithromycin as a safer drug in pregnancy. We also prescribed nitazoxanide b.i.d., vitamin C 1 gm q.d., and zinc supplements for five days. For her husband, vitamin $\mathrm{C}$ and zinc were only prescribed. A marked improvement and relief of all symptoms was reported for them within the 5-day-course. Notably, only nitazoxanide +/- azithromycin should be administered in the third trimester COVID-19 pregnant patients, without NSAIDs, to avoid premature fetal closure of the cardiac ductus arteriosus for pregnant COVID-19 patients in their third trimester [26].

\section{Managing Pediatric COVID-19 Patients}

We also used this protocol to manage four highly suspected children ( $1.5-9$ years $)$ by a history of close contact with a positive COVID-19 patient. They recovered while receiving the described five-day regimen with no reports of adverse effects.

\section{Case 28}

A female child complained of fever, severe sore throat, headache, malaise. Her relative lymphocytic count was $19.2 \%$ (normal range: 20-40\%). A colleague has already prescribed ceftriaxone, ampicillin/sulbactam, dexamethasone, and parenteral paracetamol infusion without significant improvement in her symptoms/signs except for the fever. We have immediately stopped dexamethasone and changed paracetamol to ibuprofen. A marked improvement in her symptoms was found from the first day.

\section{Case 29 and 30}

A female child of a health care professional complained of severe sore throat, severe anorexia, diarrhea, vomiting, and her sister complaining of similar yet milder manifestations while their mother administered them paracetamol and started azithromycin when the mother consulted with us. We decided to prescribe the full ibuprofen/nitazonxanide/azithromycin regimen for the two children, and they completely recovered from their symptoms in three days but continued its five days. We suggest that the relief of the severe sore throat that restored the appetite might be due to the action of both ibuprofen and azithromycin.

\section{Case 31, 32, 33}

A mother, in early $30 \mathrm{~s}$ of age, suffered from fever up to $38.5^{\circ} \mathrm{C}$, mild rhinorrhea, sore throat, dyspnea, severe bone pain, anosmia, and ageusia. Her CBC showed leucopenia of $2.4 \times 10^{3} / \mu 1$ with reduced absolute lymphocytic count $\left(1.08 \mathrm{X} 10^{3} / \mu \mathrm{l}\right)$ and a marginally high relative lymphocytic count of $45 \%$. A colleague had prescribed azithromycin, paracetamol, zinc, and vitamin $\mathrm{C}$, and she has self-administered diclofenac potassium when her bone pain was severe. We instructed continuing azithromycin for five days and replaced paracetamol with diclofenac potassium b.i.d. for three days and once daily for the other two days, where a significant improvement was reported. Notably, her husband, in mid-30s of age, has only suffered from mild cough, sore throat, headache, and ageusia, while his CBC and CRP revealed normal. However, his serum ferritin was elevated $(536.3 \mathrm{ng} / \mathrm{ml})$. We advised him to be self-isolated with his family and allowed only zinc, vitamins $C$ and $D$ to be administered. He reported shortly relief of symptoms except for ageusia. 
A few days later, their girl experienced a fever up to $38.5^{\circ} \mathrm{C}$, rhinorrhea, headache, and malaise. Her CBC showed leucocytosis (20.5 X 103/ $/ \mu 1)$, elevated relative neutrophil count of $78 \%$, reduced relative lymphocytic count of $16 \%$, normal absolute lymphocytic count $(3.28 \mathrm{X}$ $\left.10^{3} / \mu \mathrm{l}\right)$ and positive CRP titer $(12 \mathrm{mg} / \mathrm{dl})$. We prescribed weight-adjusted doses of azithromycin and nitazoxanide for three days, as well as ibuprofen for five days. A marked improvement was reported from the first day.

Notably, for other less suspected COVID-19 children, not mentioned in this manuscript, who complained of moderate troublesome cough episodes, we prescribed the locally available antitussive suppositories or syrup containing oxomemazine, guaiphenesin, sodium benzoate, and paracetamol once at night to allow a better sleep without reported adverse effects. Interestingly, expert scientists and pediatricians have also recommended the use of NSAIDs, where clinically indicated to manage COVID-19 confirmed and suspected children [27].

\section{Case 34}

A female patient in mid-30s of age. Her case was very interesting and was described in a separate manuscript [28].

\section{Discussion}

We previously suggested that combining pharmacological and pathophysiological approaches to restore the immunological interferon homeostasis might be our perfect tool to end the COVID-19 global crisis; thus, we adopted nitazoxanide/azithromycin combination for early management of COVID-19 patients [4]. Subsequently, other studies have confirmed the genetic and clinical basis that interferons could play a role in COVID-19 [29-31].

Recently, an open-label observational study recommended a combination of drugs including azithromycin and nitazoxanide for patients diagnosed early with COVID-19. Furthermore, they have opted for nitazoxanide over other drugs such as ivermectin due to the well-known more extensive demonstration of in vitro and in vivo antiviral activity, proven efficacy against other viruses in humans, and steadier safety profile [32] to be noted that this superiority of nitazoxanide over ivermectin was previously postulated by us [4].

Similarly, a small randomized, placebo-controlled trial involving mild COVID-19 patients showed that early nitazoxanide significantly eliminated the virus, as revealed by negative swabs, in $29.9 \%$ of patients in the nitazoxanide arm versus $18.2 \%$ in the placebo arm $(p=0.009)$ and reduced viral load $(\mathrm{p}=0.006)$ as compared to placebo without serious adverse events. Furthermore, $78 \%$ of patients in the nitazoxanide arm versus $57 \%$ in the placebo arm showed complete resolution of symptoms after 1-week follow-up $(p=0.048)$ [33, 34]. Moreover, A prospective study has recently suggested that early use of nitazoxanide might decrease COVID19 complications among healthcare personnel [35].

Notably, it was suggested that NSAIDs might influence COVID-19 outcomes by modulating the immune-inflammatory responses rather than modifying susceptibility to infection or viral replication. It has also been shown that NSAID treatment did not affect ACE2 expression in human cells and mice, SARS-CoV-2 entry, or replication in vitro. NSAIDs were shown to dampen the induction of proinflammatory cytokines that are upregulated by SARS-CoV-2 infection in mice [36], and we agree with their call to assess their effects in humans, while 
suggesting that the beneficial effects of NSAIDs on humoral and cellular immunity can likely be discovered [7].

Moreover, significant elevation of lymphocytes was frequently observed in most of our patients after completing our protocol and we postulate that this might be considered as a preliminary clinical proof of our suggested COVID-19 pathogenesis theory [6, 28, 37], while the significant decrease of the elevated neutrophils count in some patients might represent the efficacy of the used antibiotics in eliminating an associated secondary bacterial infection. Interestingly, the absolute lymphocyte count was shown to be an early prognostic marker associated with disease severity and other clinical outcomes in COVID-19 [38].

We were aware that our early pioneering recommendation to use NSAIDs as the first choice of COVID-19 treatment has for long been contradictory with the more common approach that preferred paracetamol. Furthermore, it is worth noting that paracetamol does not possess major peripheral anti-inflammatory effects compared to NSAIDs[40] and unfortunately, extremely unpleasant academic misconduct has led to the avoidance of NSAIDs in COVID-19 as treatment priorities. Since March 2020, we have been trying to refute these claims until we finally, six months later, we were enabled to publish a peer-reviewed article to join other colleagues [41-43]. However, NSAIDs for COVID-19 are still avoided, or at best of second choice, in many countries around the world.

Notably, other colleagues have shown aspirin, which is a non-steroidal anti-inflammatory drug, to be associated with a significantly lower rate of mechanical ventilation, intensive care unit admission, and in-hospital mortality after controlling for confounding variables [44], and we have preprinted our insight for the proper interpretation of this important study [8] and added more potential mechanisms involving in the potential benefits of NSAIDs in COVID-19 management [45].

In our study, we initially used ibuprofen to manage our COVID-19 patients after we acquitted it of the claimed potential damage [41]. However, with comparable efficacy and safety, we turned to diclofenac potassium and other nonsteroidal anti-inflammatory drugs and reserved ibuprofen for pediatric patients. Interestingly, ibuprofen use was not associated with worse COVID-19 clinical outcomes [46-51], while its use has been shown to be significantly associated with diminished COVID-19 risk for hospitalization, as revealed by an In silico cohort study that examined the electronic health records from 7,360 COVID-19 patients with positive RT-PCR test results. Furthermore, ibuprofen and naproxen were more commonly prescribed among individuals who do not need intensive care [52]. Similarly, prescribed NSAIDs were not found to be associated with COVID-19 mortality, and their administration in COVID-19 is recommended not to be affected by unproven fear [51] and another large study has also confirmed NSAIDs safety in COVID-19 management, and policymakers were recommended to review their advice for the use of NSAIDs in COVID-19 patients [55].

However, relatively few clinical trials are currently underway to test the possible role of ibuprofen in COVID-19, including a lipid ibuprofen testing that began in April 2020 with the last update in October of that year and will end in September 2021 in the UK (NCT04334629), but an Egyptian retrospective observational study tested the potential benefit of NSAIDs in the improvement of COVID-19 outcome in patients admitted to ICU, which has been labelled as complete on February 2021 (NCT04757792). 
Similarly, the updated BMJ Best Practice guidelines for COVID-19 management, on November 27, 2020, have recommended paracetamol or ibuprofen for the symptomatic management of fever and pain. Although they have clearly stated that there is no evidence of severe adverse effects of ibuprofen on COVID-19, they have also recommended that ibuprofen should be used at the lowest effective dose and for the shortest possible time to control COVID19 symptoms. We had adopted this pharmacovigilant approach in our practice long before these recommendations evolved. BMJ and NICE recommended either paracetamol or ibuprofen use for COVID-19, and we look forward to the day when paracetamol would be the second choice only when NSAIDs are absolutely contraindicated [6]. Moreover, in our study, NSAIDs were reported to be also remarkably superior to paracetamol for controlling high fever, headache, and malaise.

Importantly, an objective analysis found no evidence of a causation between ibuprofen and the risk of thromboembolism. When this risk is claimed, it is often associated with long-term and/or high-dose use [53], which is the opposite of what we have adopted. On the contrary, we claim that the anti-IL-6 properties of our adopted short course of NSAIDs may be capable of reducing the incidence of thromboembolism [7, 54].

Notably, we permitted all patients to continue the already prescribed vitamin C, vitamin D, zinc, lactoferrin, or other relatively harmless supplements. However, we have changed any already prescribed dose of zinc according to our clinical and academic judgment [17]. Therefore, we suggest that the right dose of these supplements is almost harmless, whether they might have a beneficial synergistic effect or not.

Notably, the imaging tests, including CT, showed a lack of specificity, and the PCR and SARS CoV-2 IgM tests possessed sensitivity flaws [39]. We suggest that our management protocol has a major advantage as it could be started early to manage COVID-19 suspected patients without the need for expensive/complex investigations. Most patients who received our protocol early in the COVID-19 course improved within five days compared to other patients who contacted us relatively later. Interestingly, some young individuals were close contacts to positive COVID-19 cases with no or very mild symptoms, e.g., mild sore throat or mild cough without fever. Only isolation $+/$ - zinc and vitamins were advised, and reassurance was granted during their smooth follow-up.

On the other hand, we agree that the relatively small number of patients represented in our study is a very important limitation. However, clinical case reports detect novelties and hypothesize and resolve ethical limitations [56]. Moreover, we are aware that observational studies have a known bias over interpretation tendency [56] and therefore, we presented only the most trustworthy findings. We have no conflict of interest of any sort and we repeatedly recommend performing controlled clinical trials with sufficient strength and double-blind randomization against the current standard care protocols for over a year, in vain $[4,6]$. Notably, many of our patients were being amazed when we prescribed these "cheap and easily available drugs" as they described, and we suggest that a placebo effect was almost excluded. For many patients, their experience started with negative skepticism but soon changed into deep gratitude when recovered from their COVID-19.

Furthermore, we opted not to require extensive investigations for each patient because of the lack of fund from our side to ask for and the limited financial or logistical resources of many of 
our patients. We received the provided photocopies of the performed assessments and investigations they captured using their mobile phones; thus, we could not ask for better quality photos as we knew this is the best they could offer.

However, we suggest that other researchers with better capabilities should test this protocol and other scientifically supported drugs [17] and release their results and/or modifications for the best interests of patients and all people who are eagerly anticipating these results.

\section{Conclusion}

Taken together, we wish to suggest that our described short course NSAIDs/nitazoxanide/azithromycin protocol, has shown prompt efficacy, full compliance, and excellent tolerability. Our regimen showed no serious side effects while being able to manage a wide range of COVID-19 patients safely. Moreover, we wish to suggest that the described immunomodulatory properties of our adopted drugs might also prove potentially lifesaving for other fatal viral diseases, especially when used early in their immunopathologic course [37]. We used relatively safe and FDA-approved drugs, which are readily available in most countries worldwide and are not expensive, especially for the best interests of patients in the developing countries. None of our patients, who received the described protocol, has complained of post/para COVID syndrome. We postulate improper COVID-19 pharmacotherapy as a major cause of this syndrome based on the analysis of some patients who have requested help [57]. Moreover, our analysis of discussed clinical results, coming from researchers worldwide, suggests that our early pioneering and repeatedly ignored positive recommendations for the early use of NSAIDs in COVID-19 patients might also be proved lifesaving as ours for nitazoxanide/azithromycin.

Finally, we wish to emphasize that precious lives of patients who might not promptly receive a safe/effective vaccine, especially in the developing countries, and other patients who are not permitted to receive a vaccine-like most pediatric and pregnant populations, together with those who might encounter vaccine-resistant SARS CoV-2 variants in all countries are still at stake Thus, properly designed and sufficiently powered controlled clinical trials should start soonest, another call from our side and we wish it might be finally listened to as we genuinely believe that all human lives are equal, precious, and worth saving.

\section{Funding}

None

\section{Conflict of Interests}

The author declares no competing interests.

\section{Data Availability}

The de-identified laboratory investigations of many of the described patients were published in numerous preprints. The author is available to discuss any related subject, and all the mentioned patients are in best of health and some have been treated since April 2020. 


\section{Acknowledgments}

I wish to sincerely thank all the patients mentioned in this manuscript and their family members who carefully followed their treatment. All the mentioned medical consultations were performed free of charge. All or any personal thanks should go to my Lord and Savior, Jesus Christ, and to my beloved mother, Neama Botros Rezk. Also, many thanks to Dr. Haroun Akhnoukh from Egypt and Dr. Pablo Juárez from Mexico for their precious confirmatory clinical feedback after reading some preprints of this manuscript.

\section{References}

[1] Ibarrondo FJ, Fulcher JA, Goodman-Meza D, Elliott J, Hofmann C, Hausner MA, et al. Rapid Decay of Anti-SARS-CoV2 Antibodies in Persons with Mild Covid-19. N Engl J Med, 2020;383(11):1085-1087.

[2] Kemp SA, Collier DA, Datir R, Gayed S, Jahun A, Hosmillo M, et al. Neutralising antibodies drive Spike mediated SARSCoV-2 evasion. medRxiv, 2020:2020.12.05.20241927.

[3] Kelleni M. Autoimmunity and Antibody Dependent COVID-19 Enhancement of SARS CoV-2 Vaccination: A Global Human Right to Know then Decide. Authorea (Preprint), 2021:1022541/au16212665113093279/v5.

[4] Kelleni MT. Nitazoxanide/Azithromycin combination for COVID-19: A suggested new protocol for COVID-19 early management. Pharmacol Res, 2020:104874.

[5] Kelleni M. Ibuprofen potential addition to COVID-19 early management protocols: could it be superior to paracetamol and hydroxychloroquine? Authorea (Preprint), 2020. 10.22541/au.158878847.71450719

[6] Kelleni MT. Early use of non-steroidal anti-inflammatory drugs in COVID-19 might reverse pathogenesis, prevent complications and improve clinical outcomes. Biomed \& Pharmacother, 2021;133:110982.

[7] Kelleni MT. NSAIDs/Nitazoxanide/Azithromycin Repurposed for COVID-19: Potential Mitigation of the Cytokine Storm Interleukin-6 Amplifier via Immunomodulatory Effects. Expert Rev Anti Infect Ther, 2021. 10.1080/14787210.2021.1939683.

[8] Kelleni M. Potential Crucial Role of COX-1 and/or COX-2 Inhibition, NSAIDs or Aspirin Triggered Lipoxins and Resolvins in Amelioration of COVID-19 Mortality. Authorea (Preprint), 2021. 10.22541/au.162126683.31375600/v2

[9] Kelleni MT. Tocilizumab, Remdesivir, Favipiravir, and Dexamethasone Repurposed for COVID-19: a Comprehensive Clinical and Pharmacovigilant Reassessment. SN Compr Clin Med, 2021;3(4):919-23.

[10] Pappan N, Benkhadra R, Papincak D, Ashker K, Uchin J, Sidique N, et al. Values and Limits of Telemedicine: a Case Report. SN Compr Clin Med, 2021;3, 317-319.

[11] Tan Q, Duan L, Ma Y, Wu F, Huang Q, Mao K, et al. Is oseltamivir suitable for fighting against COVID-19: In silico assessment, in vitro and retrospective study. Bioorg Chem, 2020;104:104257.

[12] Pan H, Peto R, Karim QA, Alejandria M, Henao-Restrepo AM, García CH, et al. Repurposed antiviral drugs for COVID19 -interim WHO SOLIDARITY trial results. medRxiv, 2020. 2020.10.15.20209817.

[13] Horby PW, Mafham M, Bell JL, Linsell L, Staplin N, Emberson J, et al. Lopinavir-ritonavir in patients admitted to hospital with COVID-19 (RECOVERY): a randomised, controlled, open-label, platform trial. Lancet, 2020;396(10259):1345-52.

[14] la Marca G, Barp J, Frenos S, Mugelli A, Galli L, Calistri E, et al. Thermal inactivation of SARS COVID-2 virus: Are steam inhalations a potential treatment? Life Sci, 2021;265:118801.

[15] Millar BC, Moore JE. Re-purposing of domestic steam disinfectors within the Hospital-at-Home setting: Reconciliation of steam disinfector thermal performance against SARS- CoV-2 (COVID-19), norovirus and other viruses' thermal susceptibilities. Infect Dis Health 2021;26(2):156-9.

[16] Borowiec A, Dąbrowski R, Kowalik I, Rusinowicz T, Hadzik-Błaszczyk M, Krupa R, et al. Elevated levels of d-dimer are associated with inflammation and disease activity rather than risk of venous thromboembolism in patients with granulomatosis with polyangiitis in long term observation. Adv Med Sci, 2020;65(1):97-101.

[17] Kelleni MT. Resveratrol-zinc nanoparticles or pterostilbene-zinc: Potential COVID-19 mono and adjuvant therapy. Biomed Pharmacother, 2021;139:111626.

[18] Baghaki S, Yalcin CE, Baghaki HS, Aydin SY, Daghan B, Yavuz E. COX2 inhibition in the treatment of COVID-19: Review of literature to propose repositioning of celecoxib for randomized controlled studies. Int J Infect Dis, 2020;101:2932. 
[19] Hong W, Chen Y, You K, Tan S, Wu F, Tao J, et al. Celebrex Adjuvant Therapy on Coronavirus Disease 2019: An Experimental Study. Front Pharmacol, 2020;11(1795).

[20] Wahba-Yahav AV. Chronic leukocytoclastic vasculitis associated with polycythemia vera: effective control with pentoxifylline. J Am Acad Dermatol, 1992;26(6):1006-7.

[21] Seirafianpour F, Mozafarpoor S, Fattahi N, Sadeghzadeh-Bazargan A, Hanifiha M, Goodarzi A. Treatment of COVID-19 with pentoxifylline: Could it be a potential adjuvant therapy? Dermatol Ther, 2020;33(4):e13733-e.

[22] Hu HR. Fatal Vitamin K-Dependent Coagulopathy Associated with Cefoperazone/Sulbactam: A Case Report. Drug Saf Case Rep, 2019;6(1):6.

[23] Walsh KA, Spillane S, Comber L, Cardwell K, Harrington P, Connell J, et al. The duration of infectiousness of individuals infected with SARS-CoV-2. J Infect, 2020;81(6):847-56.

[24] Li M, Chen P, Yuan Q, Song B, Ma J. Transmission characteristics of the COVID-19 outbreak in China: a study driven by data. medRxiv, 2020. 2020.02.26.20028431.

[25] Xiao AT, Tong YX, Gao C, Zhu L, Zhang YJ, Zhang S. Dynamic profile of RT-PCR findings from 301 COVID-19 patients in Wuhan, China: A descriptive study. J Clin Virol, 2020.127:104346.

[26] Koren G, Florescu A, Costei AM, Boskovic R, Moretti ME. Nonsteroidal Antiinflammatory Drugs During Third Trimester and the Risk of Premature Closure of the Ductus Arteriosus: A Meta-Analysis. Ann Pharmacother, 2006;40(5):824-9.

[27] Vosu J, Britton P, Howard-Jones A, Isaacs D, Kesson A, Khatami A, et al. Is the risk of ibuprofen or other non-steroidal anti-inflammatory drugs increased in COVID-19? J Paediatr Child Health, 2020;56(10):1645-6.

[28] Kelleni M. SARS CoV-2 Might exploit cells of the innate immune system to induce the Novel Acute Immune Dysrhythmic Syndrome (n-AIDS) and Para COVID-19 Syndrome: A Case Report. Authorea (Preprint) 2021. 10.22541/au.162126698.87303239/v2

[29] Grant RA, Morales-Nebreda L, Markov NS, Swaminathan S, Querrey M, Guzman ER, et al. Circuits between infected macrophages and T cells in SARS-CoV-2 pneumonia. Nature 2021;590, 635-641.

[30] Pairo-Castineira E, Clohisey S, Klaric L, Bretherick AD, Rawlik K, Pasko D, et al. Genetic mechanisms of critical illness in Covid-19. Nature 2020;591, 92-98.

[31] Lopez L, Sang PC, Tian Y, Sang Y. Dysregulated Interferon Response Underlying Severe COVID-19. Viruses 2020;12(12):1433

[32] Cadegiani FA, Goren A, Wambier CG, McCoy J. Early COVID-19 Therapy with Azithromycin Plus Nitazoxanide, Ivermectin or Hydroxychloroquine in Outpatient Settings Significantly Reduced Symptoms Compared to Known Outcomes in Untreated Patients. medRxiv, 2020. 2020.10.31.20223883.

[33] Rocco PRM, Silva PL, Cruz FF, Junior MACM, Tierno PFGMM, Moura MA, et al. Early use of nitazoxanide in mild Covid-19 disease: randomized, placebo-controlled trial. medRxiv 2020. 2020.10.21.20217208.

[34] Rocco PRM, Silva PL, Cruz FF, Junior MACM, Tierno PFGMM, Moura MA, et al. Early use of nitazoxanide in mild Covid-19 disease: randomised, placebo-controlled trial. Eur Respir J, 2020: 2003725.

[35] Mendieta Zerón H, Meneses Calderón J, Paniagua Coria L, Meneses Figueroa J, Vargas Contreras MJ, Vives Aceves HL, et al. Nitazoxanide as an early treatment to reduce the intensity of COVID-19 outbreaks among health personnel. World Acad Sci J 2021;3(3):23.

[36] Chen JS, Alfajaro MM, Chow RD, Wei J, Filler RB, Eisenbarth SC, et al. Nonsteroidal Anti-inflammatory Drugs Dampen the Cytokine and Antibody Response to SARS-CoV-2 Infection. J Virol, 2021;95(7):e00014-21.

[37] Kelleni MT. COVID-19, Ebola virus disease, and Nipah virus infection reclassification as novel acute immune dysrhythmia syndrome (n-AIDS): potential crucial role for immunomodulators. Immunologic Research 2021. 10.1007/s12026-021-09219-y.

[38] Wagner J, DuPont A, Larson S, Cash B, Farooq A. Absolute lymphocyte count is a prognostic marker in Covid-19: A retrospective cohort review. Int J Lab Hematol, 2020;42(6):761-5.

[39] Goudouris ES. Laboratory diagnosis of COVID-19. J Pediatr (Rio J) 2021;97(1):7-12.

[40] Anderson BJ. Paracetamol (Acetaminophen): mechanisms of action. Paediatr Anaesth, 2008;18(10):915-21.

[41] Kelleni MT. ACEIs, ARBs, ibuprofen originally linked to COVID-19: the other side of the mirror. Inflammopharmacol, 2020;28(6): 1477-80

[42] Moore N, Carleton B, Blin P, Bosco-Levy P, Droz C. Does Ibuprofen Worsen COVID-19? Drug safety 2020;43(7): 6114.

[43] Giollo A, Adami G, Gatti D, Idolazzi L, Rossini M. Coronavirus disease 19 (Covid-19) and non-steroidal antiinflammatory drugs (NSAID). Ann Rheum Dis, 2021;80(2):e12. 
[44] Chow JH, Khanna AK, Kethireddy S, Yamane D, Levine A, Jackson AM, et al. Aspirin Use Is Associated With Decreased Mechanical Ventilation, Intensive Care Unit Admission, and In-Hospital Mortality in Hospitalized Patients With Coronavirus Disease 2019. Anesth Analg, 2021;132(4).

[45] Kelleni M. NSAIDs Immunomodulation in COVID-19 Might Inhibit SARS CoV-2 ORF Proteins Induced Caspase Activation, Necroptosis and Endoplasmic Reticulum Stress. Authorea (Preprint), 2021. 10.22541/au.162126663.38675715/v3

[46] Rinott E, Kozer E, Shapira Y, Bar-Haim A, Youngster I. Ibuprofen use and clinical outcomes in COVID-19 patients. Clin Microbiol Infect, 2020;26(9):1259.e5-1259.e7.

[47] Lund LC, Kristensen KB, Reilev M, Christensen S, Thomsen RW, Christiansen CF, et al. Adverse outcomes and mortality in users of non-steroidal anti-inflammatory drugs who tested positive for SARS-CoV-2: A Danish nationwide cohort study. PLoS Med 2020;17(9):e1003308.

[48] Abu Esba LC, Alqahtani RA, Thomas A, Shamas N, Alswaidan L, Mardawi G. Ibuprofen and NSAID Use in COVID-19 Infected Patients Is Not Associated with Worse Outcomes: A Prospective Cohort Study. Infect Dis Ther, 2020;10(1): 253268.

[49] WHO (Scientific Brief). The use of non-steroidal anti-inflammatory drugs (NSAIDs) in patients with COVID-19. 2020, April 19.

[50] Abu Esba LC, Alqahtani RA, Thomas A, Shamas N, Alswaidan L, Mardawi G. Ibuprofen and NSAID Use in COVID-19 Infected Patients Is Not Associated with Worse Outcomes: A Prospective Cohort Study. Infect Dis Ther 2021;10(1):25368 .

[51] Wong AYS, MacKenna B, Morton CE, Schultze A, Walker AJ, Bhaskaran K, et al. Use of non-steroidal anti-inflammatory drugs and risk of death from COVID-19: an OpenSAFELY cohort analysis based on two cohorts. Ann Rheum Dis, 2021:annrheumdis-2020-219517.

[52] Castro VM, Ross RA, McBride SMJ, Perlis RH. Identifying common pharmacotherapies associated with reduced COVID19 morbidity using electronic health records. medRxiv, 2020. 2020.04.11.20061994.

[53] Arjomandi Rad A, Vardanyan R, Tas NR. Ibuprofen and thromboembolism in SARS-COV2. J Thromb. Haemost, 2020;18(9):2425-7.

[54] Tamaki H, Khasnis A. Venous thromboembolism in systemic autoimmune diseases: A narrative review with emphasis on primary systemic vasculitides. Vasc Med, 2015;20(4):369-76.

[55] Drake TM, Fairfield CJ, Pius R, Knight SR, Norman L, Girvan M, et al. Non-steroidal anti-inflammatory drug use and outcomes of COVID-19 in the ISARIC Clinical Characterisation Protocol UK cohort: a matched, prospective cohort study. Lancet Rheumatol, 2021;3(7):E498-E506.

[56] Nissen T, Wynn R. The clinical case report: a review of its merits and limitations. BMC Res Notes 2014;7:264.

[57] Kelleni M. SARS CoV-2 Vaccines, Remdesivir and Favipiravir Might Have Led to SARS CoV-2 B.1.617 Variants: India First but We Can Intervene. Authorea (Preprint) 2021; 10.22541/au.162126655.51131205/v6. 